\title{
RAREFIED BAND IN SPECTRUM OF TWO-MAGNON STATES OF HEISENBERG FERROMAGNET ON CLOCK DIAL PLATE
}

\author{
T. LULEK
}

Institute of Physics, A. Mickiewicz University, Matejki 48/49, 60-769 Poznań, Poland

\author{
AND A. WAL
}

Institute of Physics, Higher Pedagogical School, Rejtana 16, 35-310 Rzeszów, Poland

(Received February 1, 1995)

The Heisenberg isotropic exchange Hamiltonian with the nearest neighbour interactions for the ring of $N=12$ nodes (the clock dial plate) with the spin $1 / 2$ has been diagonalized within the manifold of all 66 two-magnon excitations from the ferromagnetic ground state. The spectrum consists of 5 full bands, i.e. bands defined over the whole Brillouin zone, and a single band which is doubly rarefied, i.e. defined only for even values of quasimomentum. The rarefied band is found to be dispersionless outside the centre of the Brillouin zone. It separates two bands of "weakly bounded" spin waves from two other which are "weakly antibounded". The last remaining band is situated below this collection of scattered spin waves, and describes two tightly bounded spin deviations. The spectrum exhibits dispersion, but it is degenerate (singlet and quintet) at the boundary of the Brillouin zone.

PACS numbers: $02.20 .-\mathrm{a}, 05.50 .+\mathrm{q}, 75 . \mathrm{Jm}, 75.30 . \mathrm{Ds}$

\section{Introduction}

Theories of energy spectra of various excitations in solids (phonons, electrons, polarons, magnons, excitons, etc.) base heavily on the notion of a band. A band $A$ is realised as a real-valued function $\varepsilon_{A}: B \rightarrow R$ over the Brillouin zone $B$, describing the dispersion law for an appropriate excitation. Thus energy levels of the system are classified by two indices: the band index $A$ and the quasimomentum $k \in B$. Such a picture implicitly assumes that each quasimomentum $k \in B$, admissible by appropriate quantization conditions (usually by Born-von Kármán periodic boundary conditions), is realised exactly once for a given non-degenerate band $A$. 
It implies, in turn, that the distribution of quantum states of the system over the Brillouin zone $B$ is completely homogeneous.

The latter feature has been definitely abandoned for the case of the Heisenberg model of a finite magnet [1-5]. For the linear magnetic chain consisting of $N$ nodes, each with the spin $s$, a combinatorial analysis unambiguously reveals that the distribution $\rho: B \rightarrow \mathcal{Z}$ of quantum states of the magnet over the Brillouin zone is inhomogeneous. The quasimomentum $k \in B$ is an exact quantum number of the Heisenberg model, resulting from its translational symmetry group $C_{N}$. Thus the integer $\rho(k)$ is the dimension of that subspace of the $(2 s+1)^{N}$-dimensional space of all quantum states of the magnet which corresponds to the irreducible representation (irrep) $k$ of the group $C_{N}$. Variation of $\rho(k)$ over the Brillouin zone $B$ implies therefore existence of rarefied bands in the energy spectrum of the magnet.

The aim of the present paper is an explicit demonstration of existence of the doubly rarefied band in the spectrum of two-magnon excitations in a finite periodic linear Heisenberg ferromagnet, consisting of $N=12$ nodes, each with the spin $1 / 2$. We derive such a rarefied band by diagonalization of the isotropic exchange Hamiltonian with the nearest-neighbours interactions within the space of all two-magnon excitations over the ferromagnetic ground state. In fact, we discuss the band structure of all two-magnon states, and localise the rarefied band just in the middle of the collection of "scattered bands", i.e. weakly bounded superpositions of two single spin waves $[6,7]$.

\section{The Heisenberg magnet on the clock dial plate}

$$
\begin{aligned}
& \text { Let } \\
& \tilde{N}=\{j \mid j=1,2, \ldots, 12\}
\end{aligned}
$$

be the set of nodes of a linear chain consisting of $N=12$ nodes, each with the $\operatorname{spin} 1 / 2$,

$$
\tilde{n}=\{i \mid i=1,2\}
$$

be the set of projections of a single-node spin, such that $i=1$ and 2 corresponds to the projection $1 / 2$ and $-1 / 2$, respectively, and

$$
\tilde{n}^{\tilde{N}}=\{f: \tilde{n} \rightarrow \tilde{N}\}
$$

be the set of all mappings from $\tilde{n}$ to $\tilde{N}$. Each such mapping denotes a magnetic configuration, and can be presented more explicitly in the form

$$
|f\rangle=\left|i_{1}, \ldots, i_{12}\right\rangle, \quad i_{j} \in \tilde{n}, j \in \tilde{N} .
$$

The set (3) of all magnetic configurations provides an orthonormal basis in the space

$$
L=\operatorname{lc}_{\mathfrak{c}} \tilde{n}^{\tilde{N}}
$$

of all quantum states of the magnet $\left(\operatorname{lc}_{\mathcal{C}} \tilde{n}^{\tilde{N}}\right.$ denotes the linear closure of the set $\tilde{n}^{\tilde{N}}$ over the field $\mathcal{C}$ of complex numbers).

Here we use another basis proposed in Ref. [5] which is essentially the basis adapted to the symmetry group $C_{N}$, which exploits the embedding $C_{N} \subset \Sigma_{N}$ into 
the symmetric group $\Sigma_{N}$ on the set $\tilde{N}$. The set $\tilde{n}^{\tilde{N}}$ splits under the action of the symmetric group $\Sigma_{N}$ into orbits $O_{\mu}$. Each such an orbit is labelled by a bipartition $\mu=\left(\mu_{1}, \mu_{2}\right)$ of $N, \mu_{i}$ denoting the occupation number for the spin projection $i \in \tilde{n}$. Under the restriction to $C_{N} \subset \Sigma_{N}$, each orbit $O_{\mu}$ splits into several orbits $O_{\mu, \kappa, \alpha}$ of the cyclic group $C_{N}$, labelled by divisors $\kappa$ of $N$ and repetition labels $\alpha$. Each orbit $O_{\mu, \kappa, \alpha}$ spans a $\bar{\kappa}$-dimensional subspace

$$
L_{\mu, \kappa, \alpha}=\operatorname{lc}_{\mathcal{C}} O_{\mu, \kappa, \alpha} \subset L
$$

of the space $L$, with

$$
\bar{\kappa}=N / \kappa
$$

being the divisor complementary to $\kappa$ in the lattice $K(N)$ of all divisors of $N$.

Each linear unitary space $L_{\mu, \kappa, \alpha}$ spanned on the orbit $O_{\mu, \kappa, \alpha}$ of the cyclic group $C_{N}$ is invariant under the action of $C_{N}$, and splits into one-dimensional irreducible subspaces labelled by the quasimomentum $k$ from the Brillouin zone $B$. The key observation, leading to the notion of a rarefied band is that

$$
\operatorname{dim} L_{\mu, \kappa, \alpha}=\bar{\kappa}=N / \kappa,
$$

so that only for the trivial divisor $\kappa=1$, the corresponding space $L_{\mu, 1, \alpha}$ is $N$-dimensional, and thus covers the whole Brillouin zone $B$

$$
B=\left\{k=0, \pm 1, \pm 2, \ldots,\left\{\begin{array}{ll} 
\pm N / 2-1, N / 2 & \text { for } N \text { even, } \\
\pm(N-1) / 2 & \text { for } N \text { odd }
\end{array}\right\}\right.
$$

which yields a full band. The case $\kappa=1$ involves orbits of the regular representation of the cyclic group $C_{N}$, in short regular orbits. All other orbits $\kappa>1$ correspond to a non-trivial stabiliser $C_{\kappa} \triangleleft C_{N}$, and consist of $\bar{\kappa}=N / \kappa<N$ configurations. The associated linear space $L_{\mu, \kappa, \alpha}$ decomposes into irreps belonging to a subset $B / \kappa \subset B$ of the Brillouin zone, given by

$$
B / \kappa=\{k=\xi \kappa \mid \xi=1,2, \ldots, \bar{\kappa}\} \subset B,
$$

and referred to as the $\kappa$-tuply rarefied Brillouin zone which yields a rarefied band.

Accordingly, the basis in $L$, adapted to the symmetry imposed by the chain $C_{N} \subset \Sigma_{N}$ of subgroups, can be written down as

$$
b=\{|\mu \kappa \alpha k\rangle \mid \mu \in P(N), \kappa \in K(N), \alpha \in \tilde{m}(\mu, \kappa), k \in B / \kappa\},
$$

where $P(N)$ is the set of all bipartitions of $N$, and

$$
\tilde{m}(\mu, \kappa)=\{\alpha=1,2, \ldots, m(\mu, \kappa)\}
$$

is the set of labels of orbits of the group $C_{N}$ with the stabiliser $C_{\kappa}, \dot{m}(\mu, \kappa)$ being the multiplicity in the decomposition

$$
R^{\Sigma_{N}: \Sigma^{\mu}} \downarrow C_{N} \approx \sum_{\kappa \in K(N)} m(\mu, \kappa) R^{N: \kappa}
$$

of the transitive representation $R^{\Sigma_{N}: \Sigma^{\mu}}$ of the group $\Sigma_{N}$ acting on the orbit $O_{\mu}$ into transitive representations $R^{N: \kappa}$ of the subgroup $C_{N} \subset \Sigma_{N}$, with $\Sigma^{\mu} \subset \Sigma_{N}$ and $C_{\kappa} \triangleleft C_{N}$ being appropriate stabilisers in the symmetric and cyclic group, respectively.

We consider in this paper an example corresponding to the orbit $O_{\mu}$ of the symmetric group $\Sigma_{12}$ with

$$
\mu=(10,2)
$$


which corresponds to $r=2$ spin deviations from the ground ferromagnetic configuration $\left|f_{\text {ferro }}\right\rangle=|11 \ldots 1\rangle$. The corresponding transitive representation with the Young subgroup

$$
\Sigma^{10,2}=\Sigma_{10} \times \Sigma_{2}
$$

decomposes under the restriction to $C_{12}$ as

$$
R^{\Sigma_{12}: \Sigma^{(10,2)}} \downarrow C_{12} \approx 5 R^{12: 1}+R^{12: 2} \text {. }
$$

Accordingly, the orbit $O_{(10,2)}$ decomposes as

where

$$
O_{(10,2)}=\bigcup_{\alpha=1}^{6} O_{\alpha}
$$

$$
O_{\alpha}=\{|p, \alpha\rangle \mid p \in \tilde{N}\}, \quad \alpha=1,2, \ldots, 6,
$$

and $|p, \alpha\rangle$ denotes such a configuration $\left|i_{1}, \ldots i_{N}\right\rangle$, for which

$$
i_{j}= \begin{cases}2 & \text { for } j=p \text { or } p+\alpha \\ 1 & \text { otherwise. }\end{cases}
$$

In other words, the label $\alpha$ of the orbit of $C_{N}$ denotes the distance between reversed spins.

The orbit $O_{(10,2)}$ of all configurations with two spin deviations splits therefore into 5 regular orbits $\alpha=1, \ldots, 5$, each having 12 elements, and a single irregular orbit $\alpha=6$, with 6 elements. Representatives of these orbits are listed in Table. The energy spectrum of two-magnon excitations should thus consist of 5 full bands, each with exactly one quantum state for each quasimomentum $k$ of the Brillouin zone, and one doubly rarefied band, with one quantum state for each even $k$, i.e. for $k=0, \pm 2, \pm 4,6$, and "kinematical vacancies" for odd quasimomenta $k=$ $\pm 1, \pm 3, \pm 5$. We proceed to show that this is indeed the result of diagonalization of the Heisenberg Hamiltonian.

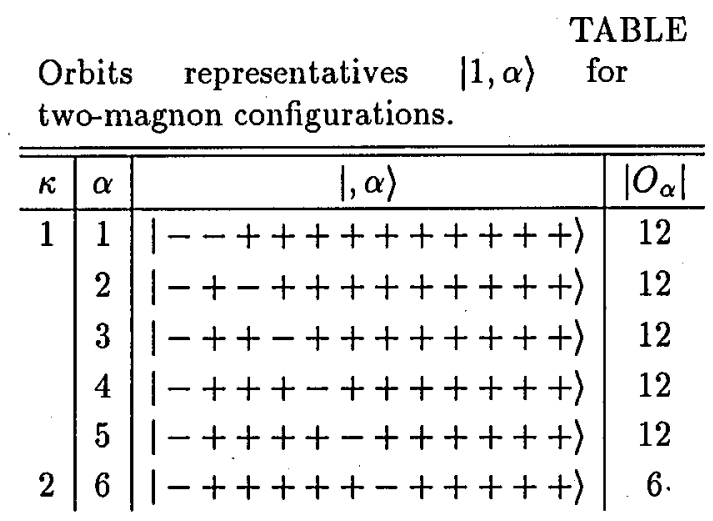

The basis states adapted to the symmetry of the translation group $C_{12}$ are usual Bloch states of the form of travelling waves

$$
|\alpha, k\rangle=\frac{1}{\sqrt{12}} \sum_{p=1}^{12} \varepsilon^{p k}|p, \alpha\rangle, \quad \alpha \in \tilde{m}(\mu, 1) \equiv\{1,2,3,4,5\}, \quad k \in B,
$$


and

$$
|6, k\rangle=\frac{1}{\sqrt{6}} \sum_{p=1}^{6} \varepsilon^{2 p k}|p, 6\rangle, \quad k \in B / 2,
$$

where $B / 2=\{0, \pm 2, \pm 4,6\}$ is the doubly rarefied Brillouin zone (cf. Eq. (10)), and $\varepsilon=\mathrm{e}^{2 \pi \mathrm{i} / 12}$

is a primitive root of unity of order $N=12$.

\section{The Heisenberg Hamiltonian and its diagonalization}

The Heisenberg Hamiltonian with the nearest-neighbour interaction is

$$
\hat{H}=J \sum_{j \in \hat{N}} \hat{s}_{j} \cdot \hat{s}_{(j+1) \bmod N}
$$

where $\hat{s}_{j}$ is the spin vector operator at the $j$-th node, and $J$ is the exchange parameter which is negative $(J<0)$ for ferromagnets. It is convenient to introduce the operators

$$
\begin{aligned}
& \hat{E}_{ \pm 1}=\sum_{j \in \bar{N}} \hat{s}_{j}^{ \pm} \hat{s}_{j+1}^{\mp}, \\
& \hat{E}_{0}=\sum_{j \in \tilde{N}} \hat{s}_{j}^{z} \hat{s}_{j+1}^{z},
\end{aligned}
$$

with $\hat{s}_{j}^{ \pm}=\hat{s}_{j}^{x} \pm \mathrm{i} \hat{s}_{j}^{y}$. These operators satisfy the following formulas:

$$
E_{ \pm 1}|p, \alpha\rangle= \begin{cases}(1 \pm 1) / 2|p-1,2\rangle+(1 \mp 1) / 2|p, 2\rangle & \text { for } \alpha=1 \\ |p, \alpha \mp 1\rangle+|p \mp 1, \alpha \pm 1\rangle & \text { for } \alpha=2, \ldots, 5 \\ |p+N / 2+(1 \mp 1) / 2, N / 2-1\rangle & \\ +|p+(1 \mp 1) / 2, N / 2-1\rangle & \text { for } \alpha=6\end{cases}
$$

and

$$
E_{0}|p, \alpha\rangle= \begin{cases}(N-4) / 4|p, 1\rangle & \text { for } \alpha=1 \\ (N-8) / 4|p, \alpha\rangle & \text { for } \alpha=2, \ldots, 6\end{cases}
$$

Clearly, the space

$$
L^{(2)}=\operatorname{lc}_{C} O_{(10,2)}
$$

of all two-magnon states is invariant under the Hamiltonian (23). The corresponding $66 \times 66$ secular matrix is diagonal with respect to quasimomentum $k$. Each even value of $k$ is associated with a 6 -dimensional secular eigenproblem, with the base labelled by $\alpha=1,2, \ldots, 6$, whereas each ocid value involves a 5 -dimensional eigenproblem with $\alpha=1,2, \ldots, 5$. Matrices $A(k)=\left\{\left\langle\alpha, k|\hat{H}| \alpha^{\prime}, k\right\rangle / J\right\}$ are given by

$$
A(k)=\left(\begin{array}{llllll}
2 & a & 0 & 0 & 0 & 0 \\
a^{*} & 1 & a & 0 & 0 & 0 \\
0 & a^{*} & 1 & a & 0 & 0 \\
0 & 0 & a^{*} & 1 & a & 0 \\
0 & 0 & 0 & a^{*} & 1 & c \\
0 & 0 & 0 & 0 & c & 1
\end{array}\right), k \in B / 2
$$


for even $k$, matrices for odd $k$ are obtained from (29) by deleting the last row and column. Here,

$$
J a=\langle\alpha, k|\hat{H}| \alpha+1, k\rangle=\frac{1}{2}\left(1+\varepsilon^{-k}\right), \quad \alpha \in \tilde{m}(\mu, 1) \backslash 5
$$

is the off-diagonal matrix element within the regular orbits, and

$$
J c=\langle 5, k|\hat{H}| 6, k\rangle \begin{cases}\sqrt{2} & \text { for } k=0, \\ 0 & \text { otherwise }\end{cases}
$$

corresponds to hybridization between the regular orbit $\alpha=5$ and the irregular one $\alpha=6$.

Equation (31) implies that the eigenproblem for matrices $A(k)$ is essentially six-dimensional for the centre $k=0$ of the Brillouin zone only, whereas outside the centre it splits according to

$$
A(k)=A^{f}(k) \oplus A^{r}(k), \quad k \in B, k \neq 0,
$$

where $A^{f}(k)$ is the five-dimensional matrix, corresponding to regular orbits, and

$$
A^{r}(k)= \begin{cases}1 & \text { for } k \in\{ \pm 2, \pm 4,6\} \subset B, \\ 0 & \text { otherwise }\end{cases}
$$

is the one-dimensional eigenproblem, related immediately to the irregular orbit $\mathrm{O}_{6}$. It follows that the associated rarefied band is

$$
\varepsilon^{r}(k)=\left\{\begin{array}{cl}
0.759 J & \text { for } k=0 \\
J & \text { for } k \in\{ \pm 2, \pm 4,6\}
\end{array}\right.
$$

i.e. it is dispersionless outside the centre of the Brillouin zone. The corresponding eigenstates for $k=0$ are given by Eq. (21).

We proceed to point out the position of the doubly rarefied band (33) within the whole manifold of two-magnon excitations. An interesting particular case is provided by the "boundary" $k=6$ of the discrete Brillouin zone $B$. Equation (29) implies that the non-diagonal matrix element vanishes for this case, and thus the matrix $A(6)$ given by Eq. (29) becomes diagonal. The label $\alpha \in \tilde{m}(\mu, \kappa)$, $\kappa=1,2$, denoting the separation of reversed spins in the chain $\tilde{N}$, becomes thus an additional exact quantum number at the boundary $k=6$ of the Brillouin zone. The corresponding energies are

$$
\varepsilon^{\alpha}(6)=\left\{\begin{array}{cl}
2 J & \text { for } \alpha=1, \\
J & \text { for } \alpha \in\{2,3,4,5,6\} .
\end{array}\right.
$$

Thus the energy $\varepsilon^{1}(6)=2 J$ is non-degenerate, and corresponds to the travelling wave of two nearest-neighbour spin deviations (represented by the first row of Table). The corresponding eigenstate reads (cf. Eq. (20))

$$
|1,6\rangle=\frac{1}{\sqrt{12}} \sum_{p=1}^{12}(-1)^{p}|p, 1\rangle .
$$

Prolongation of this state to the whole Brillouin zone yields the finite analogy of the band of two bounded spin waves $[6,7]$. 


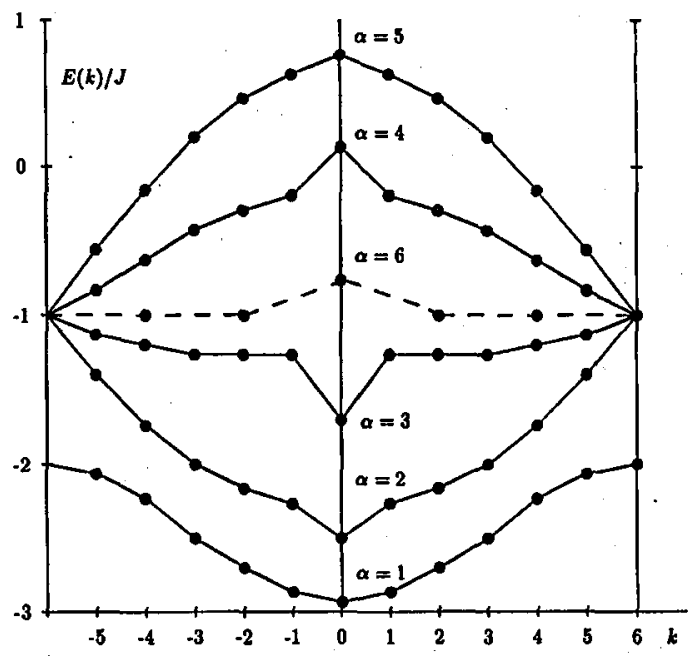

Fig. 1. Bands of two-magnon excitations in the clock dial plate. Heavy dots denote the eigenvalues $\epsilon^{\alpha}(k)$ of the Heisenberg Hamiltonian. Continuous lines $(\alpha=1,2,3,4,5)$ denote the full bands, and the dotted line $(\alpha=6)$ - the doubly rarefied band. The band $\alpha=1$ corresponds to two strongly bounded spin waves. The centre $k=0$ of this band is degenerated with the ground level energy of the ferromagnet. The other bands form a collection of scattered spin waves, with "weakly bounded" bands $\alpha=2$ and 3 , and "weakly antibounded" $\alpha=4$ and 5, separated by the doubly rarefied band $\alpha=6$. The latter band is dispersionless outside the centre $k=0$. All scattered bands $(\alpha \neq 1)$ are degenerate at the boundary $k=6$ of the Brillouin zone. Observe that there is no eigenvalue of the Hamiltonian for odd values $k= \pm 1, \pm 3, \pm 5$ of quasimomentum for the doubly rarefied band $\alpha=6$.

Energies ${ }^{\dagger} \varepsilon^{\alpha}(6)=J, \alpha \in\{2,3,4,5,6\}$ are degenerate at the boundary of the Brillouin zone. One of them, namely $\varepsilon^{\alpha}(6)=\varepsilon^{r}(6)$, can be readily recognised as that belonging to the rarefied band (cf. Eq. (33)). The other four, $\alpha \in\{2,3,4,5\}$, give rise to full bands, two of them lying above $(\alpha \in\{2,3\})$ and the two other ( $\alpha \in\{4,5\}$ ) - below the dispersionless rarefied band. All these five bands (four full and one doubly rarefied) form the finite analogy of the quasicontinuous spectrum of scattered two-magnon excitations. In particular, they are well separated from the band of bounded spin waves, and lie above this band. It is. interesting to observe that all full bands exhibit, contrary to the rarefied band, an appreciable dispersion which vanishes only at the boundary of the Brillouin zone, and increases when passing to the centre (Fig. 1).

\section{Final remarks and conclusions}

We performed an exact numerical diagonalization of the Heisenberg exchange Hamiltonian for the finite ring of $N=12$ nodes with the spin $1 / 2$ and nearest-neighbour interactions, within the manifold of all two-magnon excitations from the ferromagnetic ground state. The 66-dimensional space spanned by this man- 
ifold splits into 6 energy bands. 5 bands are regular, i.e. each of them is defined over the whole Brillouin zone, whereas one is doubly rarefied, defined only for even values of the quasimomentum $k$. An exact diagonalization of the Hamiltonian matrix (29), exploiting the exact quantum number $k \in B$ and orbit structure of the set $O_{(10,2)}$ of all magnetic configurations with two spin deviations, allows us to formulate some general conclusions concerning the nature of eigenfunctions corresponding to these bands.

(i) We have determined the sets

$$
s p(k)=\left\{\epsilon^{s}(k)\right\}, \quad k \in B
$$

of all eigenvalues of each matrix $A(k), k \in B$, corresponding to the Heisenberg Hamiltonian (23) within the subspace $L^{(2)}$ of all two-magnon excitations, together with the corresponding eigenfunctions

$$
|s, k\rangle=\sum_{\alpha} C_{\alpha s}(k)|\alpha, k\rangle .
$$

The eigenproblem of this Hamiltonian yields 5 (6) eigenvalues for each odd (even) quasimomentum $k$, i.e.

$$
|s p(k)|= \begin{cases}5 & \text { for } k \in\{ \pm 1, \pm 3, \pm 5\} \\ 6 & \text { for } k \in\{0, \pm 2, \pm 4,6\}\end{cases}
$$

and

$$
\sum_{k \in B}|s p(k)|=66 .
$$

The label $s$ in Eq. (37) denotes consecutive eigenvalues, ordered in accordance with increase in energy within each set $s p(k)$, i.e. $s=1, \ldots, 5(6)$ for $k$ odd (even). Such labelling of eigenvalues $\epsilon^{s}(k)$ and corresponding eigenfunctions $|s, k\rangle$ are unambiguous everywhere outside the boundary $k=6$ of the Brillouin zone $B$, since all these eigenvalues are non-degenerate within each set $s p(k), k \neq 6$. An ambiguity appears in the case of the boundary set $s p(6)$ which consists of the singlet $\epsilon^{(6)}$ and the quintet $\epsilon^{s}(6), s=2, \ldots, 6$, of degenerate states $|s, 6\rangle$. In this case, the diagonalization implies that

$$
C_{\alpha 1}(6)= \begin{cases}.1 & \text { for } \alpha=1 \\ 0 & \text { otherwise }\end{cases}
$$

which means that the eigenstate of the singlet $\epsilon^{1}(6)$ coincides with the state $|1,6\rangle$ given by Eq. (36), and corresponding to the orbit $\alpha=1$ of deviations of the nearest neighbours. Also, the degenerate eigenstates of the quintet can be chosen to coincide with some states $|\alpha, 6\rangle$ of an appropriate orbit of $\alpha$-th neighbours.

(ii) There arises a question about the relation between the discrete set

$$
\operatorname{spec}\left(H, L^{(2)}\right)=\cup_{k \in B} s p(k)
$$

of eigenvalues of two-magnon excitations of the finite crystal, and the band structure of the infinite one. A simplified form of this question reads: how to arrange elements of the set (42) into energy bands? Fortunately in our case a simple extension of criterion of increase in energy from each set $s p(k), k \in B$, to the whole Brillouin zone yields band structure shown in Fig. 1. One easily resolves 5 full 
bands denoted by continuous lines, and a single doubly rarefied band denoted by the dotted line. We find therefore that, in general, the finite set (42) of eigenvalues can be resolved reasonably into bands.

(iii) Numerical results of diagonalization show that in the interior

$$
B^{\prime}=B \backslash\{0,6\}
$$

of the Brillouin zone $B$ each band $s$ is characterised uniquely by a value of the label $\alpha$ of neighbourhood of reversed spins such that the coefficient $C_{\alpha s}(k)$ in Eq. (38) is prevailing for each $k \in B^{\prime}$. The bands can be therefore labelled by $\alpha$ in a genealogical way. This labelling is shown in Fig. 1.

(iv) The labelling by $\alpha$ described in (iii) can be extended to the boundary $k=6$ of the Brillouin zone, but it definitely breaks at the centre $k=0$. Here, the values of $\alpha$ with prevailing $C_{s \alpha}(0)$ are $1,3,4,2$, and 5 for $s=2,3,4,5$, and 6 , respectively. In particular, the eigenstate corresponding to the lowest eigenvalue

$$
\epsilon^{1}(0)=-3 J
$$

is

$$
|s=1, k=0\rangle=(2 \cdot 3)^{-1(2)} \sum_{\alpha=1}^{6}|\alpha, 0\rangle,
$$

i.e. is completely symmetric with respect to the neighbourhood label $\alpha$, and thus does not make any discrimination between full and rarefied orbits. On the other hand, the irregular orbit $\alpha=6$ does not label any band at the centre $k=0$ of the Brillouin zone, but is rather "dissolved" over all 6 energy levels.

(v) All bands labelled by regular orbits, i.e. $\alpha \in \tilde{m}(\mu, 1)$, exhibit an appreciable dispersion of energy, due to interference effects implied by the non-diagonal matrix elements $a$ of Eq. (30) (this element is constant within the whole stratum of regular orbits) and $c$ of Eq. (31) (between the regular orbit $O_{5}$ and irregular $O_{6}$ ). The largest dispersion corresponds to the centre $k=0$ of the Brillouin zone. It involves also the rarefied band $\alpha=6$. The dispersion decreases for $|k|$ increasing, and becomes the smallest at the boundary. The rarefied band $\alpha=6$ is dispersionless outside the centre of the Brillouin zone, and thus its eigenfunctions are completely specified by the irregular orbit $O_{6}$ for $k \neq 0$.

(vi) The band $\alpha=1$ is naturally recognised as that of two bounded spin waves. It corresponds to the case when the reversed spin are nearest neighbours each of the other. At the centre $k=0$ of the Brillouin zone this band achieves the energy $-3 J$, which is degenerate with that of the ferromagnetic ground configuration, and corresponds therefore to two Goldstone bosons. The other cases, $\alpha=2,3,4,5,6$, yield a collection of bands corresponding to scattered spin waves. In particular, the rarefied band $\alpha=6$ is one of them. It separates the "weakly bounded" bands $\alpha=4$ and 5 from "weakly antibounded" bands $\alpha=2$ and 3 .

(vii) We have demonstrated the existence of the rarefied band $\alpha=6$, on the basis of exact diagonalization. Somehow peculiar "kinematical vacancies", corresponding to odd quasimomenta $k= \pm 1, \pm 3, \pm 5$, can be understood by observing that the corresponding irregular orbit $O_{6}$ involves only 6 magnetic configurations which are able to cover only doubly rarefied Brillouin zone $B / 2=\{0, \pm 2, \pm 4,6\}$, and cannot span any state corresponding to odd quasimomentum. 
(viii) It is worth to mention that recent progress on quantum electronics in nanoscopic scale, in perticular magnetic quantum dots [8], creates a real chance for an immediate detection of such finite size effects as rarefied bands, even and odd quasimomenta, and other arithmetic features of small systems.

\section{Acknowledgment}

This work was supported with the grant No. 202389101 of the State Committee for Scientific Research (Republic of Poland).

\section{References}

[1] T. Lulek, J. Phys. (France) 45, 29 (1984).

[2] W. Florek, T. Lulek, J. Phys. A 20, 1921 (1987).

[3] B. Lulek, in: Symmetry and Structural Properties of Condensed Matter, Eds. W. Florek, T. Lulek, M. Mucha, World Sci., Singapore 1991, p. 131.

[4] B. Lulek, Acta Phys. Pol. B 22, 371 (1991).

[5] B. Lulek, J. Phys., Condens. Matter. 4, 8737 (1992).

[6] M. Wortis, Rhys. Rev. 132, 85 (1963).

[7] D.C. Mattis, The Theory of Magnetism, Harper and Row, New York 1965.

[8] D. Loss, D.P. deVincenzo, G. Grinstein, J.S. Smith, Physica B 189, 189 (1993). 